This item was submitted to Loughborough's Research Repository by the author.

Items in Figshare are protected by copyright, with all rights reserved, unless otherwise indicated.

\title{
Chiral phase states of the Hubbard Hamiltonian
}

PLEASE CITE THE PUBLISHED VERSION

PUBLISHER

(C) American Physical Society

LICENCE

CC BY-NC-ND 4.0

REPOSITORY RECORD

Kusmartsev, F.V., and M.L. Ristig. 2019. "Chiral Phase States of the Hubbard Hamiltonian". figshare. https://hdl.handle.net/2134/1304. 


\title{
Chiral phase states of the Hubbard Hamiltonian
}

\author{
F. V. Kusmartsev and M. L. Ristig \\ Institut für Theoretische Physik, der Universität zu Köln, D-5000 Köln 41, Germany \\ and L. D. Landau Institute for Theoretical Physics, Moscow, GSP-1, Kosygina 2, V-334, U.S.S.R.
}

(Received 28 January 1991)

\begin{abstract}
We present a variational approach different from that based on Gutzwiller's ansatz by investigating chiral flux-phase states of the Hubbard Hamiltonian in analogy to the treatment of the fractional quantum Hall effect. The proposed class of generalized Laughlin trial functions is specialized to permit detailed consideration of a set of states that includes a ferromagnetic ground state generated by a spontaneous gauge field in conjunction with a fictitious magnetic field having a flux commensurate with the filling. We evaluate the trial energy expectation values and demonstrate that the treatment is, at least, appropriate for the Hubbard model with sufficiently large on-site Coulomb repulsion and low electron densities. The members of the special set of trial states may be suitably classified by the flux quanta of the associated field and may be characterized either by integer or fractional quantum numbers. The excitations designated by fractional quantum numbers, which are not commensurate with the filling, are identified as flux-phase states breaking the symmetries of the lattice.
\end{abstract}

An understanding of the ground state of a strongly correlated electron system may perhaps come from improved studies of the ground-state properties of the Hubbard model and of its excitations. Anderson ${ }^{1}$ proposed the concept of resonant valence-bond states in which the many-body system is viewed as a Bose liquid of singlet or triplet pairs of fermions bound to a lattice. Reference 2 provides a study of such states within a mean-field description.

Alternative studies are based on the hypothesis that the ground state may be generated by electrons which condense on a two-dimensional lattice and carry spontaneous orbital currents. ${ }^{3,4}$ This idea may be developed by introducing so-called flux-phase states which break the discrete translational symmetry and parity of the lattice. ${ }^{5-8}$ Such an approach leads to a pointlike Fermi surface, ${ }^{4}$ a locally relativistic Dirac spectrum, and interesting transport phenomena. For example, the optical resistivity decreases linearly with decreasing temperature. ${ }^{9,10}$

Various flux-phase states have been studied for rather simple two-dimensional models and for a Hubbard model with SU(n) symmetry. ${ }^{4}$ Here, we shall concentrate on the standard two-dimensional Hubbard Hamiltonian. Our analysis is based on the assumption that the groundstate correlations and excitations of the model are driven by the presence of a spontaneous gauge field with magnetic length $l_{0}$ and a fictitious magnetic field $B$. This premise connects our study with Laughlin's treatment of the fractional quantum Hall effect. ${ }^{11}$ Therefore we are particularly interested in the properties of the Hubbard Hamiltonian for large on-site Coulomb repulsion, $U \rightarrow \infty$. Within the context of the Hubbard model, we employ a set of suitably correlated wave functions of a generalized Laughlin form. For simplicity, the wave functions are adapted to a $L \times L$ lattice, the associated one-body density matrices being required to obey periodic boundary conditions. The members of the set of trial wave functions may be properly specified by the total flux through an elementary lattice plaquette generated by the gauge and fictitious fields. The total flux is quantized and may carry either integer multiples or fractions of an elementary quantum $\Phi_{e}$. These states are chiral flux states. They break the lattice symmetries, parity $P$, translation $T$, charge conjugation $C$, and the composite symmetries except CPT symmetry, which is still conserved.

For large Coulomb repulsion we may specialize the set of correlated wave functions and evaluate analytically the corresponding energy expectation values of the Hubbard Hamiltonian. The calculated energies represent upper bounds on the ground-state energy and are independent of the on-site Coulomb repulsion. The lowest energy is attained for the state with zero or integer total flux. This is a ferromagnetic state in which the flux of the fictitious magnetic field is commensurate with the filling. This state has not been investigated before. The chiral flux states with fractional total flux represent excitations and may be separated into two branches, one branch with nonnegative energies and another branch with zero energy for which the states are infinitely degenerate.

We begin with an extended version of the Hubbard Hamiltonian,

$$
H=-t \sum_{\langle i, j\rangle, \sigma} a_{i \sigma}^{\dagger} a_{j \sigma}+U \sum_{i} n_{i}+n_{i}+V \sum_{\langle i, j\rangle, \sigma} n_{i \sigma} n_{j \sigma},
$$

involving as parameters the electron hopping integral $t$, the on-site repulsive Coulomb potential $U$, and an additional potential $V$. The parameter $V$ measures the Coulomb-and/or polarization-potential between electrons on neighboring lattice sites. The operator $a_{i \sigma}^{\dagger}\left(a_{i \sigma}\right)$ creates (destroys) an electron with spin projection $\sigma$ $\left(\sigma=+\right.$ or - ) at a lattice site $i$, and $n_{i \sigma}$ is the occupation number operator $a_{i \sigma}^{\dagger} a_{i \sigma}$. The summations in Eq. (1) extend over the lattice sites $i$ or-as indicated by $\langle i, j\rangle, \sigma$ - over all distinct pairs of nearest-neighbor sites, along with the spin projection $\sigma$.

The Hamiltonian (1) models a system of $N+$ electrons with spin projection $\sigma=+$ and $N$ - electrons with $\sigma=-$. For simplicity, we limit ourselves to a study of the impor- 
tant case $N_{-}=N_{+} \equiv N$ and we assume that the electrons are uniformly distributed over a $L \times L$ square lattice with lattice constant $a$. The Pauli exclusion principle restricts the electron density $\rho=N / L^{2}$ to values $\rho \leq 1$.

Let us first evaluate the ground-state energy corresponding to the Hamiltonian (1). It is given by the standard expression

$$
\begin{aligned}
E= & -t \rho \sum_{\langle i, j\rangle, \sigma} n\left(z_{i \sigma}, z_{j \sigma}\right)+U \rho^{2} \sum_{i} g\left(z_{i+}, z_{i}-\right) \\
& +V \rho^{2} \sum_{\langle i, j\rangle, \sigma} g\left(z_{i \sigma}, z_{j \sigma}\right) .
\end{aligned}
$$

Equation (2) involves the one-body density matrix $n\left(z_{i \sigma}, z_{j \sigma^{\prime}}\right)$ and the two-body density-matrix elements $g\left(z_{i \sigma}, z_{j \sigma^{\prime}}\right)$. These quantities are functions of the coordinates $z_{i}=x_{i}+i y_{i}, z_{j}=x_{j}+i y_{j}$ of the lattice sites $i, j$ on which an electron or electrons of the given spin projection $\sigma$ are located. The matrix elements are defined by the standard multidimensional integrals over the probability distribution associated with the chosen many-body wave function. ${ }^{12}$ These quantities are normalized such that $n\left(z_{i \sigma}, z_{i \sigma}\right)=1$ and $g\left(z_{i \sigma}, z_{j \sigma^{\prime}}\right) \rightarrow 1$ as $\left|z_{i \sigma}-z_{j \sigma^{\prime}}\right| \rightarrow \infty$.

The microscopic treatment of the fractional quantum Hall effect by Laughlin ${ }^{11}$ offers a fruitful prescription for tailoring a set of correlated wave functions that may approximately describe the ground state and excited states of the Hubbard Hamiltonian (1). In analogy to the electrodynamic gauge field driving the Hall mechanism, we assume the occurrence of a spontaneous gauge field that generates the correlations present in the eigenstates of the Hubbard Hamiltonian and is responsible for the orbital currents on the lattice. This picture motivates the trial ansatz

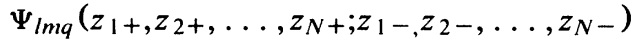

$$
\begin{aligned}
& =N_{\operatorname{lmq}} \prod_{1=i<j}^{N_{+}}\left(z_{i+}-z_{j+}\right)^{l} \prod_{1=i<j}^{N_{-}}\left(z_{i-}-z_{j-}\right)^{m} \prod_{i, j=1}^{N_{+}, N_{-}}\left(z_{i+}-z_{j-}\right)^{q} \prod_{i=1}^{N_{-}} \Psi\left(z_{i-}\right) \prod_{i=1}^{N_{+}} \Psi\left(z_{i+}\right),
\end{aligned}
$$

for approximate representation of the ground state and excited states of the Hubbard model. Expression (3) generalizes the familiar Laughlin form of wave function. The factor $N_{l m q}$ normalizes the function (3) to unity. To ensure complete antisymmetry of ansatz (3), the exponents $l$ and $m$ must be odd integers, but at this point the exponent $q$ may be any non-negative number. The single-particle wave function entering ( 3 ) is

$$
\Psi(z)=\exp \left(-\frac{|z|^{2}}{4 l_{0}^{2}}-i \frac{\phi_{B}}{4}\right),
$$

wherein the magnetic length $l_{0}$ characterizes the effect of a spontaneous gauge field ${ }^{11}$ inducing orbital currents on the lattice. The additional phase $\phi_{B} / 4$ is associated with a fictitious magnetic field $B$ given by $\phi_{B}=2 \int A d s$, where the gauge $A=(-y, x, 0) B$ is used.

The parameters $B, l_{0}, l, m$, and $q$ may be chosen optimally to minimize the ground-state energy (2) with respect to trial function (3). Many-body techniques are available ${ }^{12-14}$ for evaluating the density-matrix elements needed in Eq. (2). Postponing such numerical calculations for the complete set of parametrized functions (3), we may test the quality of the proposed description by focusing on the properties of Hamiltonian (1) for large on-site Coulomb repulsion at low electron densities. In the limiting regime defined by $U \rightarrow \infty$ and $\rho \rightarrow 0$, the associated ground-state energy may be exactly calculated in analogy to the treatment of Refs. 15 and 16, with the result

$$
E=-8 t \rho L^{2} .
$$

In this particular case an adequate specialization of (3) is provided by $l=m=q=1$, a choice of which permits analytic evaluation of the requisite one- and two-body density-matrix elements. ${ }^{17}$ In the thermodynamic limit
$(N \rightarrow \infty$, density $\rho$ held constant $)$, the results are

$$
\begin{gathered}
n\left(z_{i \sigma}, z_{j \sigma^{\prime}}\right)=\exp \left(\frac{1}{4 l_{0}^{2}}\left(2 z_{i \sigma^{\prime}} z_{j \sigma^{\prime}}^{*}-\left|z_{i \sigma}\right|^{2}-\left|z_{j \sigma^{\prime}}\right|^{2}\right)\right. \\
\left.-\frac{i}{2} \int_{i}^{j} A d s\right) \\
g\left(z_{i \sigma}, z_{j \sigma^{\prime}}\right)=1-\exp \left(-\left|z_{i \sigma}-z_{j \sigma^{\prime}}\right|^{2} / 2 l_{0}^{2}\right) .
\end{gathered}
$$

To match the electron distribution properly to the square $L \times L$ lattice, Laughlin's relation ${ }^{11}$

$$
\rho=\frac{a^{2}}{4 \pi l_{0}^{2}}
$$

between the density $\rho$ and the magnetic length $l_{0}$ must hold.

Inserting Eqs. (6)-(8) into Eq. (2) and performing the lattice summations we arrive at the intermediate result

$$
E=-8 t \rho L F(\Phi) \exp (-\pi \rho)+4 V \rho^{2} L^{2}[1-\exp (-2 \pi \rho)]
$$

for the trial energy. The function $F(\Phi)$ is defined by

$$
F(\Phi)=\sum_{l=1}^{L} \cos \left(\frac{l \Phi}{2}\right),
$$

where $\Phi$ is the value of the total flux through an elementary plaquette of area $a^{2}$,

$$
\Phi=\frac{a^{2}}{l_{0}^{2}}-B a^{2}
$$

Following Ref. 4, the latter expression may be obtained by extracting the phases of the one-body density matrix (6). For example, in the $x$ direction the phase corresponding to 
the link $\langle i, i \pm 1\rangle$ of lattice site $i$ is

$$
\theta_{i j}= \pm\left(\frac{1}{2 l_{0}^{2}}-\frac{B}{2}\right) x_{i} a \text {. }
$$

Calculating the sum of the relevant phases by going around the links of the elementary plaquette in the clockwise direction, we arrive at the result (11) for the flux per plaquette.

To proceed further, we impose periodic (toroidal) boundary conditions on the phase of the one-body density matrix appropriately matched to the square lattice. ${ }^{18}$ This implies a quantization of the total flux (11) according to $\Phi_{n}=4 n \pi / L$ or $\Phi_{n}=4 n \pi /(L+2)$, with $n=0,1,2, \ldots$ The quantized fluxes may be separated into three different classes,

$$
\Phi_{n}=\left\{\begin{array}{l}
n \Phi_{e}, n=0,1,2, \ldots, \\
\left(\frac{n}{L}+k\right) \Phi_{e}, n=0,1,2, \ldots, L-1, \\
\left(\frac{n}{L+2}+k\right) \Phi_{e}, n=0,1,2, \ldots, L+1,
\end{array}\right.
$$

where $k$ is an integer and $\Phi_{e}=4 \pi$ is the elementary quantum. The first branch is characterized by an integral quantum number $n$, and the second and third branches by fractional quantum numbers $n / L$ and $n /(L+2)$, respectively. Consequently, the function $F(\Phi)$ is quantized through conditions (13), leading to the respective cases

$$
F(\Phi)=\left\{\begin{array}{l}
L, \\
0, \\
-[1+\cos (\Phi / 2)] .
\end{array}\right.
$$

Correspondingly, we may divide the expectation values (9) for the Hubbard Hamiltonian into three branches. The states described by integral multiples of the elementary quantum $\Phi_{e}$ give an energy per plaquette of

$$
E_{1} / L^{2}=-8 \rho t \exp (-\pi \rho)+4 V \rho^{2}[1-\exp (-2 \pi \rho)] .
$$

The states with fractional quantum numbers $\Phi$ yield energies per plaquette of

$$
E_{2} / L^{2}=4 V \rho^{2}[1-\exp (-2 \pi \rho)]
$$

and

$$
\begin{aligned}
E_{3} / L^{2}= & (8 t \rho / L) \exp (-\pi \rho)[1+\cos (\Phi / 2)] \\
& +4 V \rho^{2}[1-\exp (-2 \pi \rho)]
\end{aligned}
$$

for the second and third branches, respectively. A value of $\Phi$ for the third branch is to be inserted from (13) into (17).

From Eqs. (15)-(17) it is seen that the lowest upper bound to the exact ground-state energy of Hamiltonian (1) is given by the first branch of (13). The corresponding result (15) for the energy per plaquette is independent of the total number of flux quanta $n$. The other branches give the energies (16) and (17) assigned to a set of excited states that are described as fractional flux-phase states.
At small densities $(\rho \rightarrow 0)$ expression (15) correctly reproduces the exact result (5) for the ground-state energy. At larger filling factors expression (5) represents an upper bound to the true ground-state energy. However, we are convinced that the proposed wave function (3) is well suited to provide an appropriate physical description of the ferromagnetic domain of the $(U, \rho)$ phase diagram of the Hubbard model yielding energy expectation values reasonably close to the true energies. Ansatz (5), for instance, is more flexible than a standard Gutzwiller wave function. ${ }^{19}$ It mimics the Gutzwiller projection mechanism but allows, moreover, the existence of vortices by employing the magnetic length $l_{0}$ and the fictitious magnetic field $B$ as additional variational parameters. We therefore expect that the energy expectation values with respect to ansatz (3) are lower than the corresponding Gutzwiller expectation values. The agreement of result (15) with result (5) in the limit of small filling factors and the above considerations support the view that the general ansatz (3) provides a class of many-body wave functions well suited to describing the eigenstates of Hamiltonian (1), even if the density is not so small and the Coulomb strength $U$ is not limited to the strongly repulsive region. The principal features of our results are not affected if the lattice structure is changed. The detailed forms of the function $F(\Phi)$ and of the flux quantization condition depend, of course, on the actual choice of lattice symmetry. However, replacing the square lattice by a triangular one, for example, does not affect the classification of the energies and states into those with integer and fractional quantum numbers.

The nature of the quantum states we have constructed may be further elucidated by determining their symmetry properties and their spin polarization. In particular, do the trial states (3), when specialized to the case $N_{+}=N_{-}$ and the parameter choice $l=m=q=1$, describe a polarized or an unpolarized system? This question may be answered by evaluating the expectation values of the total spin components $M_{x}$ and $M_{y}$ parallel to the lattice plane and of the component $M_{z}$ orthogonal to it. The component $M_{x}$ is calculated from

$$
\sum_{i, \sigma}\left\langle\Psi\left|S_{i \sigma}^{x}\right| \Psi\right\rangle=\sum_{i}\left\langle\Psi\left|\left(a_{i}^{\dagger}+a_{i-}+a_{i}^{\dagger}-a_{i+}\right)\right| \Psi\right\rangle,
$$

i.e., from the sum of site-diagonal elements of the onebody density matrix (6) for unlike spin projections. The result is $M_{x}=N$. The components $M_{y}$ and $M_{z}$ vanish. Thus, we find complete spin alignment parallel to the lattice plane and conclude that the fermions form a ferromagnetic phase. This finding is somewhat reminiscent of Nagaoka's result. ${ }^{20}$ We stress, however, that fluxphase states (3) describe a ferromagnetic state that is very different from the usual ferromagnetic state, by virtue of the existence of orbital currents. States of this type have not been explored before. It is apparent, of course, that the microscopic approach to the Hubbard Hamiltonian proposed here differs essentially from treatments of the paramagnetic and antiferromagnetic phases of the Hubbard model based, for example, on the Gutzwiller ansatz. ${ }^{19}$

The ferromagnetic flux-phase states generate spontane- 
ous currents and diamagnetic moments which, effectively, screen the electron-electron spin correlations. The magnetic length of the associated gauge field plays the role of a Debye radius. The fractional flux-phase states explicitly considered above can be further characterized by their symmetry properties. In contrast to the commensurate state, the flux-phase states break the symmetries of the underlying square lattice. This may be easily checked for parity $P$, translation $T$, charge conjugation $C$, and their pairwise products. However, PCT symmetry is maintained. We remark that analogous symmetry-breaking states in antiferromagnets have been predicted by Wiegmann. ${ }^{7}$

The present study gives a deeper understanding of the relationship between the properties of the Hubbard model and the mechanism of the fractional quantum Hall effect. It leads to a ground state described as a new ferromagnet- ic commensurate flux-phase state. Our analysis also leads naturally to an alternate class of excitations in twodimensional ferromagnetic systems, associated with fractional flux-phase states. In the next stage of this approach, one should proceed to a broader exploration of the properties and the consequences of wave-function ansatz (3), allowing $N_{-}$to differ from $N_{+}$. Moreover, by optimizing the parameters $l, m$, and $q$, we may study the effects of genuine dynamical correlations. This would permit a proper comparison with results of other many-body approaches incorporating spatial correlation effects, notably the Gutzwiller procedure.

We thank V. L. Pokrovsky, F. D. M. Haldane, and John W. Clark for useful discussions. This work has been supported by the A. v. Humboldt foundation.
'P. W. Anderson, Science 235, 1252 (1987).

${ }^{2}$ G. Baskaran, Z. Zou, and P. W. Anderson, Solid State Commun. 63, 973 (1987).

${ }^{3}$ V. Kalmeyer and R. B. Laughlin, Phys. Rev. Lett. 59, 2095 (1987).

${ }^{4}$ I. Affleck and J. B. Marston, Phys. Rev. B 37, 3774 (1988).

${ }^{5}$ X. G. Wen, F. Wilczek, and A. Zee, Phys. Rev. B 39, 11413 (1989).

${ }^{6}$ P. W. Anderson, Phys. Scr. T27, 60 (1989).

${ }^{7}$ P. B. Wiegmann, Phys. Scr. T27, 160 (1989).

${ }^{8}$ E. J. Mele, Phys. Rev. B 38, 8940 (1988).

${ }^{9}$ M. J. Rice, Y. R. Wang, and E. J. Mele, Phys. Rev. B 40, 5304 (1989).

${ }^{10}$ F. V. Kusmartsev and A. M. Tsvelik, Pis'ma Zh. Eksp. Teor. Fiz. 42, 207 (1985) [JETP Lett. 42, 257 (1985)].

${ }^{11}$ T. Chakraborty and P. Pietilainen, in The Fractional Quantum Hall Effect, edited by K. v. Klitzing (Springer, Berlin,
1988), and references cited therein.

${ }^{12}$ E. Feenberg, Theory of Quantum Fluids (Academic, New York, 1969).

${ }^{13}$ J. W. Clark, in Progress in Particle and Nuclear Physics, edited by D. Wilkinson (Pergamon, Oxford, 1979), Vol. 2.

${ }^{14}$ M. L. Ristig, in From Nuclei to Particles, Proceedings of the International School of Physics "Enrico Fermi," Course LVII, Varenna, 1981, edited by A. Molinari (North-Holland, Amsterdam, 1982).

${ }^{15}$ S. A. Trugman and S. Kivelson, Phys. Rev. B 31, 5280 (1985).

${ }^{16}$ V. L. Pokrovsky and A. L. Talapov, J. Phys. C 18, L691 (1985).

${ }^{17}$ B. Jancovici, Phys. Rev. Lett. 46, 386 (1981).

${ }^{18}$ C. N. Yang, Rev. Mod. Phys. 34, 694 (1962).

${ }^{19}$ M. C. Gutzwiller, Phys. Rev. Lett. 10, 159 (1963).

${ }^{20}$ Y. Nagaoka, Phys. Rev. 147, 392 (1966). 